\title{
Thermophysical and Magnetic Properties of Magnetite - Polyethylene Composite
}

\author{
N. M. Bugaev ${ }^{1}$, Ekaterina L. Kuznetsova ${ }^{1}$, Kyaw Ye Koㄹ, \\ ${ }^{1}$ Moscow Aviation Institute (National Research University), \\ Moscow, Volokolamskoe shosse, 4, 125993, \\ Russia \\ ${ }^{2}$ Defense Services Technological Academy (DSTA), \\ Mandalay-Lashio Highway Mandalay Division, Pyin Oo Lwin, \\ Myanmar
}

Received: March 6, 2021. Revised: July 30, 2021. Accepted: September 6, 2021. Published: September 9, 2021.

\begin{abstract}
In this work, it is shown that the advantage of using matrix-stabilized magnetic nanoparticles to obtain polymer nanocomposites based on them is that such nanoparticles retain their dispersion and stability of size and shape in the technological modes of obtaining polymer nanocomposite materials, and thus ensured stable ferroand superparamagnetic properties of the obtained target products.

For the production of films by the method of hot pressing from blanks obtained in an injection molding machine or a mechanochemical mixture, a manual electrically heated hydraulic press was used. The magnetic properties of nanocomposite samples (about $50 \mathrm{mg}$ on average) were studied using a vibration magnetometer.

The character of the dependence of the magnetization on the magnitude of the magnetic field confirms the ferromagnetic character of the behavior of the obtained nanocomposites. The resulting film nanocomposites exhibit ferromagnetic properties at room temperature.
\end{abstract}

Keywords-Composites, polymers, thermophysical properties, magnetite.

\section{INTRODUCTION}

A MONG the wide range of investigated nanoscale materials for various environmental and biomedical applications, magnetic nanoparticles have received considerable attention due to their intrinsic magnetic properties, making them successful as magnetically reduced catalysts, drug delivery agents, anti-cancer materials, magnetic resonance imaging, etc. This class of nanomaterials includes metallic, bimetallic nanoparticles, metal oxides, ferrites, and superparamagnetic iron oxide nanoparticles. Magnetic nanoparticles and nanocomposites have generated significant scientific and technological interest due to their potential applications in biomedicine, information technology, magnetic resonance imaging, catalysis, telecommunications, and environmental restoration [1]-[14]. Magnetic nanocomposites usually contain magnetic nanoparticles embedded in a non-magnetic or magnetic matrix. However, magnetic nanoparticles dispersed in composites usually have a strong tendency to form agglomerates to reduce the energy associated with the high surface area to volume ratio of nanosized particles [15-14]. To avoid aggregation of magnetic nanoparticles, protection strategies have been developed to chemically stabilize unprotected magnetic nanoparticles by grafting or coating with organic species, including surfactants or polymers, or coating with an inorganic layer such as silicon dioxide or carbon. Combining these functionalized magnetic nanoparticles in a polymer or other matrices to develop magnetic nanocomposite materials has proven to be more efficient [25]-[32]. There are mainly four types of magnetic nanocomposites, i.e. inorganic core nanocomposites, self-assembled nanocomposites, silicabased magnetic nanocomposites, and organic-inorganic nanocomposites [33]-[50]. Among them, organic inorganic magnetic nanocomposites have become more interesting due to the combination of the unique properties of the organic and inorganic components in one material. Hybrid organic inorganic magnetic nanocomposite materials can be obtained in situ, ex situ, microwave exposure, coprecipitation, melt mixing, ceramic glass treatment, and plasma polymerization methods [51]-[66].

Modern composites have not only a wide range of physical and mechanical properties, but are also capable of 
directionally changing them, for example, increasing fracture toughness, regulating rigidity, strength, and other properties. These possibilities are expanded when fibers of different nature and geometry are used in composites, i.e., when creating hybrid composites. In addition, these materials are characterized by the appearance of a synergistic effect (coordinated joint action of several factors in one direction).

The properties of the interface or interfacial zone, first of all, the adhesive interaction between the fiber and the matrix, determine the level of properties of composites and their retention during operation. Local stresses in the composite reach their maximum values just near or directly at the interface, where material destruction usually begins. The interface must have certain properties to ensure efficient transfer of the mechanical load from the matrix to the fiber. The adhesion bond at the interface should not be destroyed under the action of thermal and shrinkage stresses arising from the difference in the temperature coefficients of linear expansion of the matrix and fiber or as a result of chemical shrinkage of the binder during its curing.

Magnetic nanoparticles can act as a new class of non-toxic and effective flame retardants. $\mathrm{Fe}_{3} \mathrm{O}_{4}$ enhanced both thermal stability and flame retardant properties of polyvinyl alcohol. Nanoparticles were synthesized by a simple precipitation reaction without using an inert atmosphere at room temperature [33]-[50]. The nanoparticles exhibited ferromagnetic behavior at room temperature. To obtain a magnetic nanocomposite, $\mathrm{Fe}_{3} \mathrm{O}_{4}$ nanoparticles were added to polyvinyl alcohol. Dispersed nanoparticles play the role of a magnetic barrier layer, which slows down the volatilization of the product and prevents the penetration of oxygen into the sample during polymer decomposition. However, these effects for polymer-nanoparticles composites often remain unclear and additional studies are needed for better elucidation of the phenomena observed.

The aim of this study was to synthesize the composite materials based on iron oxide nanoparticles and polyethylene and characterize their magnetic properties.

\section{SYNTHESIS AND STUDY OF THE PROPERTIES OF NANOCOMPOSITES}

Iron oxide nanoparticles (II, III) (10 g) were synthesized by the reaction of coprecipitation of aqueous solutions of iron (II) and (III) chlorides in the presence of alkali in an argon atmosphere according to the following procedure: To a solution of $8.9 \mathrm{~g}(0.033 \mathrm{~mol}) \mathrm{FeCl}_{3} \cdot 6 \mathrm{H}_{2} \mathrm{O}$ and $2.1 \mathrm{~g}(0.0165$ mol) $\mathrm{FeCl}_{2} \cdot 4 \mathrm{H}_{2} \mathrm{O}\left(>97 \%\right.$, Aldrich), in water at $40{ }^{\circ} \mathrm{C}$ and vigorous stirring, concentrated $\mathrm{NH}_{4} \mathrm{OH}(25 \%)$ was added for 10-15 min., the reaction mixture was kept for $30 \mathrm{~min}$. The formed black color precipitate was washed with water until neutral reaction, separated with a static magnet or centrifugation for $15 \mathrm{~min}$, washed with additional ethyl alcohol and stored in dry benzene. A thermoplastic polymer was used as a matrix: Linear Low-Density Polyethylene (LLDPE).
For the production of films by the method of hot pressing from blanks obtained in an injection molding machine or a mechanochemical mixture, a manual electrically heated hydraulic press was used. The calculated amount of the composite material was loaded into a flat brass mold within the bounding frame of $100 \times 100 \mathrm{~mm}$ with a thickness of $0,2 \mathrm{~mm}$ for the manufacture of samples for measurements on the dielectric constant and physical and mechanical tests. Sample weight $(\mathrm{m})$, in grams, was calculated by the formula: $\mathrm{m}=\mathrm{V}(\mathrm{x} * \rho \mathrm{pe}+\mathrm{y} * \rho \mathrm{me}) * 100 \%$,

where $\mathrm{V}$ - shape volume, $\mathrm{cm}^{3}$; $\mathrm{x}$ and $\mathrm{y}$ - fractions of sample components, $\% ; \rho_{\mathrm{pe}}$ and $\rho_{\mathrm{me}}$ - density of polymer and metal filler, $\mathrm{g} / \mathrm{cm}^{3}$.

To assess the interphase layer at the interface between the particles of iron oxide and a matrix containing linear lowdensity polyethylene, the above relation is used:

$$
\left.h(t)=K_{0} \exp \left\lfloor-\frac{E \pm \kappa \sigma_{k k}}{2 R T}\right\rfloor \sqrt{t}, \quad h(t) \equiv \delta(t){ }^{*}\right)
$$

where $\mathrm{K}_{0}$ - this is a preexponential parameter determined experimentally, $\mathrm{E}$ - activation energy of the growth process of the reaction zone, $\mathrm{R}=8,314$ - universal gas constant, $\mathrm{T}$ process temperature on the Kelvin scale, $\sigma_{k k}$ - ball tensor. The parameter $\mathrm{k}$ is determined according to the experimental data.

After determining the value $\mathrm{k}$ you can establish the dependence of the thickness of the interphase layer on the holding time.

In Figure 1, such a dependence is plotted:

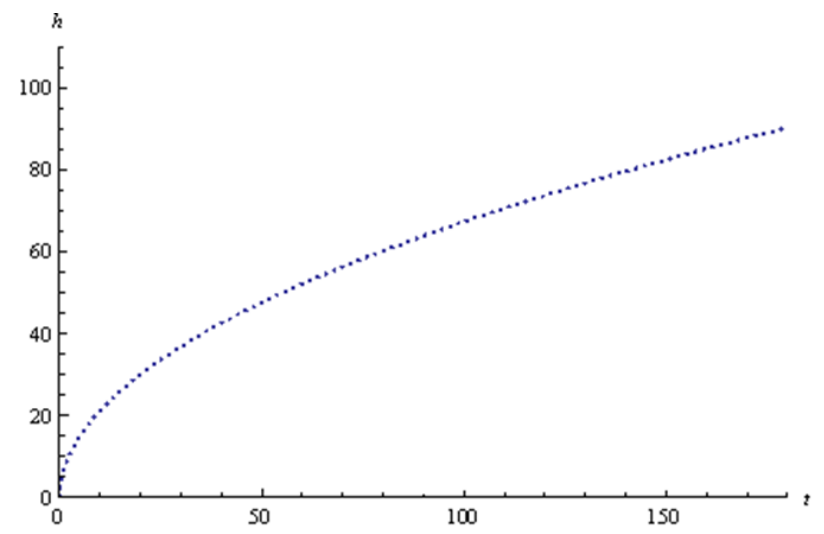

Fig. 1 Dependence of the interfacial layer thickness in nanometers.

Further the influence of pressure and temperature on the rate of formation of the interphase layer is investigated.

It was found that in the specified range of these characteristics, the growth of the interphase layer does not depend on them. This is demonstrated in Figure 2, which shows the dependences for the interfacial layer thicknesses when the parameter $\mathrm{k}$ is fixed:

The upper curve is plotted for temperature $\mathrm{T}=1020 \mathrm{~K}$, and the lower one is for $\mathrm{T}=720 \mathrm{~K}$. 


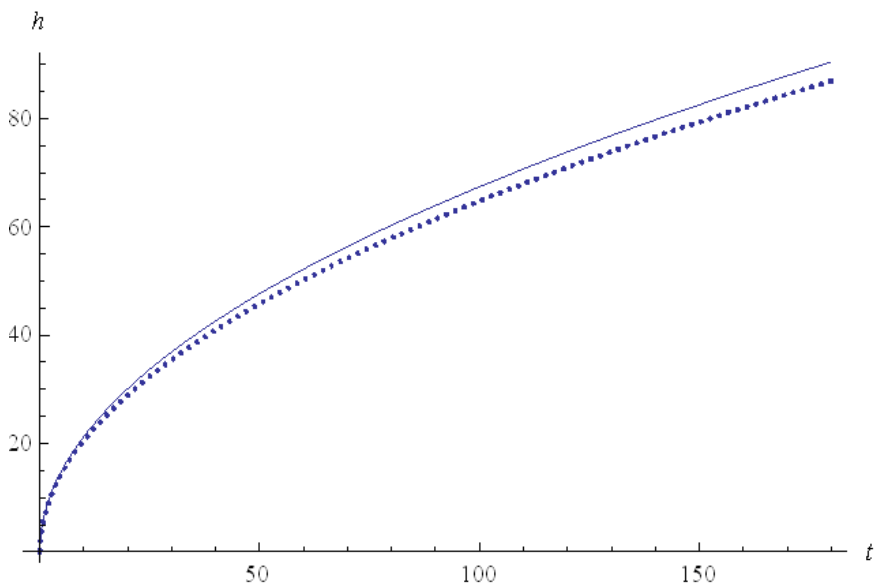

Fig. 2 Assessment of the effect of temperature on the thickness of the interfacial layer (in nanometers).

Let us find out whether the accuracy of determining the activation energy affects the estimates of the interfacial layer thickness. To do this, first the parameter $\mathrm{k}$ is determined again, according to the experimental test, and then the dependence of the layer thickness on the holding time is plotted.

After that, the time dependences for the interphase layer were constructed. The results are shown in the Figure 3:

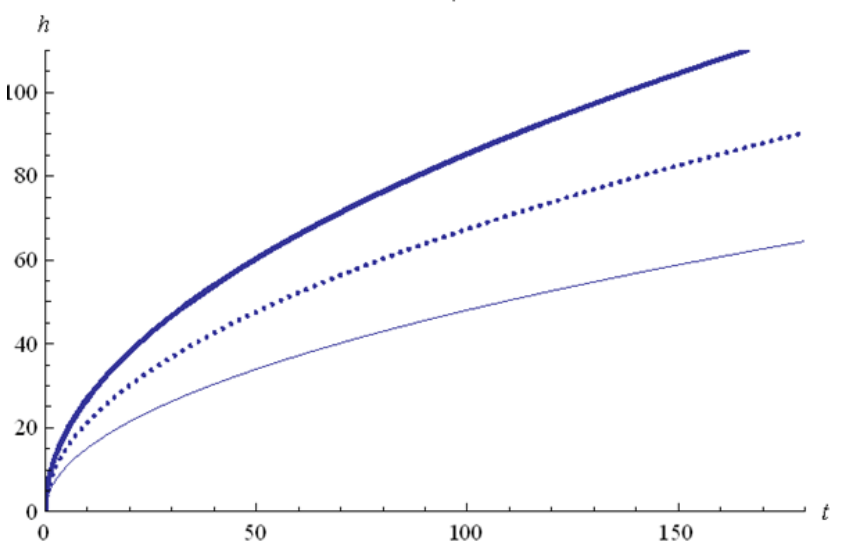

Fig. 3 Influence of the accuracy of determining the parameter $\mathrm{K}_{0}$ on the prediction of the interfacial layer thickness.

It is important to note that the accuracy of the forecast is affected by the accuracy of determining the parameters in the formula $(*)$. Therefore, it is very important to expand the experimental data, which can be used to determine (and refine) the parameters included in $(*)$. Having these data and solving the problem of identifying the parameters of the model $(*)$ by minimizing the target function (error of the theoretical dependence) in the rate selected after testing, we can significantly refine the forecast data for the thickness of the interphase layer.

According to some sources, the growth of the spinel interphase zone can begin only after the passage of the "incubation" period, which for temperatures amounts to 1000 $\mathrm{K}-2000 \mathrm{~s}$ and for temperatures above $1000 \mathrm{~K}$ decreases to
$500 \mathrm{~s}$. Thus, the studied processing time is $3 \mathrm{~min}$. may not be enough to start the growth of interfacial zones in the composite. Figure 4 shows the refined dependence of the thickness of the interfacial zone, depending on the temperature of the process and taking into account the incubation period. Here it was assumed that the incubation time decreases linearly with an increase in temperature from 970 to $1020 \mathrm{~K}$ from an initial value of $2000 \mathrm{~s}$ to a value of $500 \mathrm{~s}$.

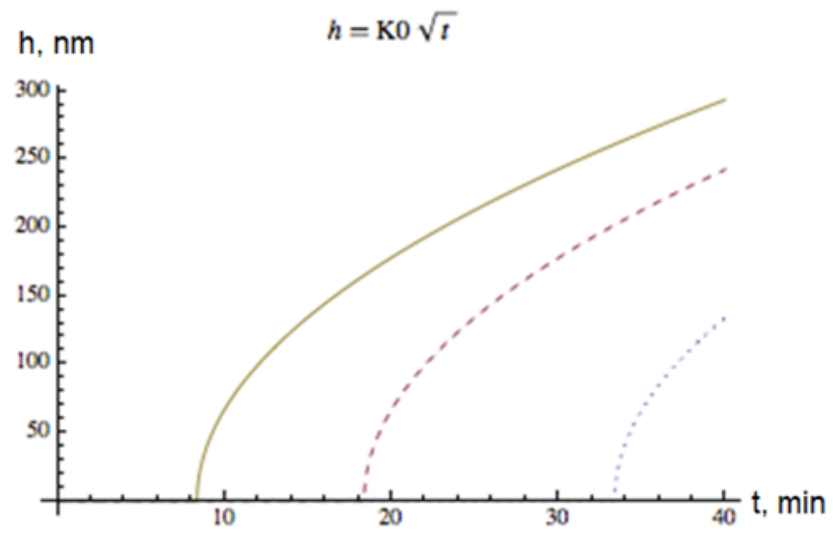

Fig. 4 Dependence of the thickness of the interphase zone on time, taking into account the incubation period (solid line $\mathrm{T}=1020 \mathrm{~K}$, dashed line $-\mathrm{T}=1000 \mathrm{~K}$, dotted line $-\mathrm{T}=970 \mathrm{~K}$ ).

Modeling shows that in a given range of temperature and pressure variation, an interfacial layer thickness of $60-120 \mathrm{~nm}$ can be realized. At the same time, neither temperature nor pressure (in the specified ranges) has a significant effect on the thickness. Optimum thickness can only be achieved by changing the holding time. Based on the preliminary calculations (which may require clarification during the experiments), it is possible to recommend carrying out the technological process for obtaining samples at a temperature of $750 \mathrm{~K}$ to ensure the shortest time of the incubation period of growth. The pressure should be minimal in the selected range $30 \mathrm{~atm}$., to increase the growth rate of the interphase zone. The process time should be 10-20 minutes to obtain an interface thickness of 80-150 nm. If the phenomenon of the incubation period of the beginning of the growth of the interfacial zone is not confirmed, the holding time should be 3-5 minutes.

\section{RESULTS AND DISCUSSION}

The magnetic properties of nanocomposite samples (about $50 \mathrm{mg}$ on average) were studied using a vibration magnetometer (VSM) M4500 EG \& G PARC, which was calibrated using a standard pure nickel sample $(90 \mathrm{mg})$ with a relative accuracy of $1 \times 10^{3}$, at room temperature. During the experiments, the magnetic field was varied from 0 to $10 \mathrm{kOe}$ at room temperature, which made it possible to measure the saturation magnetization (Ms), remanent magnetization (Mr), and coercive force $(\mathrm{Hc})$ for each sample.

The introduction of magnetite nanoparticles is reflected in the structural state of nanocomposites. 


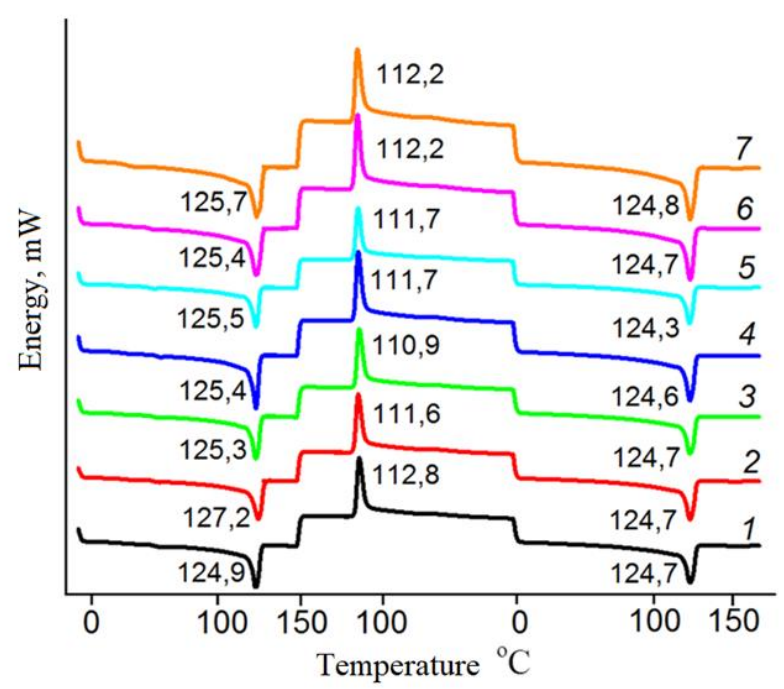

Fig. 5 DSC curves of LLDPE (1) and nanocomposites Fe3O4/LLDPE with filler concentration (wt\%): 0.5 (2), 1 (3), 3 (4), 5 (5), 10 (6), 20 (7).

DSC data (Fig. 5) indicate a systematic decrease in the enthalpy of melting $\Delta \mathrm{H}$ with an increase in the content of magnetite nanoparticles in the LLDPE polymer matrix, a similar character of the dependence is observed in the values of the degree of crystallinity of nanocomposites calculated by the formula:

$$
\chi=\mathrm{H}_{\mathrm{m}} / \mathrm{H}_{100} * 100 \%,
$$

where $\mathrm{Hm}$ - heat released during the melting of the sample, H100 - heat released during melting of $100 \%$ crystalline polymer (for LLDPE H100 = $285 \mathrm{~J} / \mathrm{g}$ ). The decrease in the degree of crystallinity of the filled polyethylene in comparison with LLDPE (Table 1) is probably associated with the formation of a less ordered crystal structure.

Table 1. Temperature, heat of fusion and degree of crystallinity nanocomposite based on $\mathrm{Fe}_{3} \mathrm{O}_{4} /$ LLDPE.

\begin{tabular}{|c|c|c|c|}
\hline \multirow{2}{*}{ Sample } & \multicolumn{3}{|c|}{$\mathrm{Fe}_{3} \mathrm{O}_{4} /$ LLDPE } \\
\cline { 2 - 4 } & $\mathrm{tm},{ }^{\circ} \mathrm{C}$ & $\Delta \mathrm{Hpl}, \mathrm{J} / \mathrm{g}$ & $\chi, \%$ \\
\hline LLDPE & 124.7 & 119 & 42 \\
\hline $0 . \mathrm{PE}$ & 124.7 & 132 & 46 \\
\hline $1 \mathrm{Fe}_{3} \mathrm{O}_{4} \mathrm{PE}$ & 124.7 & 115 & 40 \\
\hline $3 \mathrm{Fe}_{3} \mathrm{O}_{4} \mathrm{PE}$ & 124.6 & 109 & 38 \\
\hline $5 \mathrm{Fe}_{3} \mathrm{O}_{4} \mathrm{PE}$ & 124.3 & 107 & 37 \\
\hline $10 \mathrm{Fe}_{3} \mathrm{O}_{4} \mathrm{PE}$ & 124.7 & 105 & 36 \\
\hline $20 \mathrm{Fe}_{3} \mathrm{O}_{4} \mathrm{PE}$ & 124.8 & 95 & 33 \\
\hline
\end{tabular}

Magnetoactive polymer films obtained in the $\mathrm{Fe}_{3} \mathrm{O}_{4} / \mathrm{LLDPE}$ system. Magnetic characteristics (saturation magnetization
$(M s)$, remanent magnetization $(M r)$ and coercive force $(H c)$ of the obtained nanocomposites are presented in Table 2.

Table 2. Magnetic properties of polymer nanocomposite materials.

\begin{tabular}{|l|l|l|l|}
\hline Sample & $\mathrm{Ms}, \mathrm{emu} / \mathrm{g}$ & $\mathrm{Mr}, \mathrm{emu} / \mathrm{g}$ & $\mathrm{Hc}, \mathrm{Oe}$ \\
\hline $1 \mathrm{Fe}_{3} \mathrm{O}_{4} \mathrm{PE}$ & $0.32 \pm 0.04$ & $0.025 \pm 0.001$ & $44 \pm 3$ \\
\hline $3 \mathrm{Fe}_{3} \mathrm{O}_{4} \mathrm{PE}$ & $0.15 \pm 0.03$ & $0.007 \pm 0.0014$ & $29 \pm 2$ \\
\hline $10 \mathrm{Fe}_{3} \mathrm{O}_{4} \mathrm{PE}$ & $4.43 \pm 0.4$ & $0.28 \pm 0.03$ & $37 \pm 3$ \\
\hline $20 \mathrm{Fe}_{3} \mathrm{O}_{4} \mathrm{PE}$ & $8.84 \pm 0.9$ & $0.54 \pm 0.02$ & $36 \pm 2$ \\
\hline
\end{tabular}

For comparison, note that at room temperature bulk magnetite is a soft magnetic material with a coercive force $\mathrm{Hc}$ $\sim 200-400$ Oe and a saturation magnetization of $92 \mathrm{emu} / \mathrm{g}$. As can be seen from the data in the table, the magnetic properties are determined by the content of the filler, which can be clearly seen from the graphical data of the dependence of the magnetization on the strength of the applied magnetic field. The relatively small coercive force is due to the fact that the small size of nanoparticles (7-15 nm), which have superparamagnetic properties, make a significant contribution to the magnetic properties. It can also be seen that the saturation magnetization increases as the nanofiller is added.

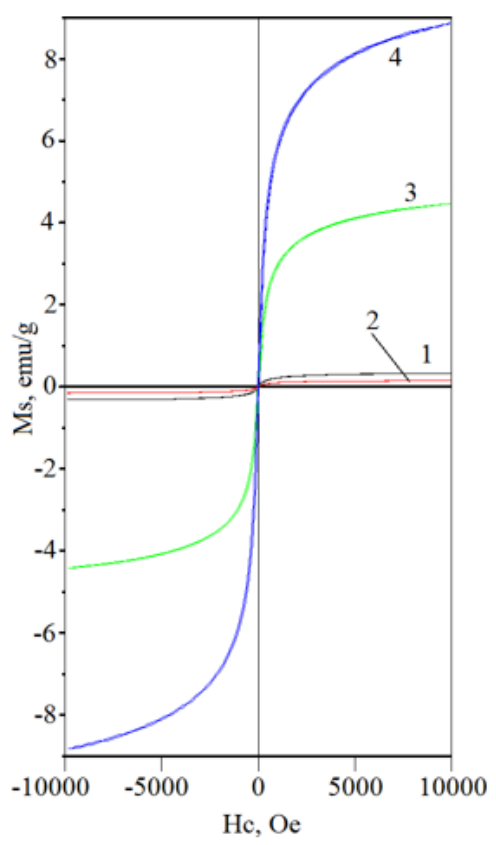

Fig. 6 Dependence of magnetization on the magnetic field, measured at $300 \mathrm{~K}$, for nanocomposite films $\mathrm{Fe}_{3} \mathrm{O}_{4} / \mathrm{LLDPE}$ on the nanofiller content: 1 (1), 3 (2), 10 (3), 20 wt.\% (4).

The character of the dependence of the magnetization on the magnitude of the magnetic field confirms the ferromagnetic character of the behavior of the obtained nanocomposites at room temperature. During the experiments, the magnetic field was varied from 0 to $10 \mathrm{kOe}$ at room temperature, which made it possible to measure the saturation magnetization (Ms), 
remanent magnetization $(\mathrm{Mr})$, and coercive force $(\mathrm{Hc})$ for each sample.

\section{CONCLUSION}

Thus, the advantage of using matrix-stabilized magnetic nanoparticles to obtain polymer nanocomposites based on them is that such nanoparticles retain their dispersion and stability of size and shape in the technological modes of obtaining polymer nanocomposite materials, and thus stable ferro- and superparamagnetic properties of the obtained target products are provided. The resulting film nanocomposites exhibit ferromagnetic properties at room temperature. As can be seen from the data obtained, the magnetic properties are determined by the content of the filler, which can be clearly seen from the graphical data of the dependence of the magnetization on the strength of the applied magnetic field. The relatively small coercive force is due to the fact that the small size of nanoparticles (7-15 nm), which have superparamagnetic properties, make a significant contribution to the magnetic properties. It can also be seen that the saturation magnetization increases as the nanofiller is added.

\section{REFERENCES}

[1] M. Sha, Y.A. Utkin, O. V. Tushavina, P.F. Pronina, "Experimental studies of heat and mass transfer from tip models made of carbon-carbon composite material $(\mathrm{cccm})$ under conditions of high-intensity thermal load" Periodico Tche Quimica, vol.17, i. 35, pp 988-997, 2020.

[2] P.F. Pronina, O.V. Tushavina, E. I. Starovoitov, "Study of the radiation situation in moscow by investigating elastoplastic bodies in a neutron flux taking into account thermal effects," Periodico Tche Quimica, 17(35), pp. 753-764, 2020.

[3] A. A. Orekhov, Y.A. Utkin, P.F. Pronina, "Determination of deformation in mesh composite structure under the action of compressive loads," Periodico Tche Quimica, 17(35), p. 599-608, 2020.

[4] A. V. Babaytsev, L. N. Rabinskiy, K. T. Aung, "Investigation of the contact zone of a cylindrical shell located between two parallel rigid plates with a gap," INCAS Bulletin, 12(Special Issue), pp. 43-52, 2020.

[5] V. G. Dmitriev, O. V. Egorova, E. I. Starovoitov, "Particularities of mathematical modeling of deformation processes for arched and panel designs of composites with large displacements and rotation angles," INCAS Bulletin, 12(Special Issue), pp. 53-66, 2020.

[6] O. V. Egorova, E. I. Starovoitov, "Non-stationary diffraction problem of a plane oblique pressure wave on the shell in the form of a hyperbolic cylinder taking into account the dissipation effect," INCAS Bulletin, 12(Special Issue), pp. 67-77, 2020.

[7] O. V. Tushavina, "Coupled heat transfer between a viscous shock gasdynamic layer and a transversely streamlined anisotropic half-space," INCAS Bulletin, 12 (Special Issue), pp. 211-220, 2020.

[8] O. A. Pashkov, "Influence of Polymer Coatings on the Mechanical Properties of Steel Samples in Tensile and
Bending Tests," Turkish Journal of Computer and Mathematics Education (TURCOMAT), vol. 12, no. 5, pp. 542-548, 2021.

[9] O. A. Pashkov, "Investigation of the Effect of Steel Plate Size and Elevated Temperature on Critical Load in Stability Tests," Turkish Journal of Computer and Mathematics Education (TURCOMAT), vol. 12, no. 10, pp. 1657-1663, 2021.

[10] Y. Sun, O. V. Egorova, E. L. Kuznetsova, "Identification of the front angle of a plane acoustic oblique pressure wave on convex surfaces with the use of analytical solution," Journal of the Balkan Tribological Association, 27(2), pp. 189-197, 2021.

[11] O. A. Pashkov, "Theoretical calculation of the thickness of interphase zones in the Al-Al2O3 composite," Turkish Journal of Computer and Mathematics Education (TURCOMAT), vol. 12, no. 10, pp. 1672-1677, 2021.

[12] O. A. Pashkov, "Experimental and Theoretical Study of Mechanical Properties of Matrix Composite Materials," Turkish Journal of Computer and Mathematics Education (TURCOMAT), vol. 12, no. 10, pp. 1678-1684, 2021.

[13] S. Vakhneev, E. Starovoitov, "Damping of circular composite viscoelastic plate vibration under neutron irradiation," Journal of Applied Engineering Science, 18(4), pp. 699-704, 2020.

[14] O. A. Butusova, "Design and Properties of Magnetically Controlled Sorbents," Turkish Journal of Computer and Mathematics Education (TURCOMAT), vol. 12, no. 5, pp. 515-519, 2021.

[15] O. A. Butusova, "Application of Magnetically Controlled Sorbents for Detoxication," Turkish Journal of Computer and Mathematics Education (TURCOMAT), vol. 12, no. 5, pp. 520-524, 2021.

[16] V. A. Pogodin, L. N. Rabinskii, S. A. Sitnikov, "3D Printing of Components for the Gas-Discharge Chamber of Electric Rocket Engines," Russian Engineering Research, vol. 39, no. 9. pp. 797-799, 2019.

[17] L. E. Kuznetsova, V. G. Fedotenkov, "Dynamics of a spherical enclosure in a liquid during ultrasonic cavitation," Journal of Applied Engineering Science, 18(4), pp. $681-686,2020$.

[18] A. V. Makarenko, E. L. Kuznetsova, "Energy-Efficient Actuator for the Control System of Promising Vehicles," Russian Engineering Research, 39(9), pp. 776-779, 2019.

[19]E. L. Kuznetsova, A. V. Makarenko, "Mathematic simulation of energy-efficient power supply sources for mechatronic modules of promising mobile objects," Periodico Tche Quimica, 15(Special Issue 1), pp. 330338, 2018.

[20] Y. Li, A. M. Arutiunian, E. L. Kuznetsova, G. V. Fedotenkov, "Method for solving plane unsteady contact problems for rigid stamp and elastic half-space with a cavity of arbitrary geometry and location," INCAS Bulletin, 12(Special Issue), pp. 99-113, 2020.

[21]M. O. Kaptakov, "Effect of Thin Polymer Layers on Mechanical Properties of Metal Surfaces," Turkish Journal of Computer and Mathematics Education (TURCOMAT), vol. 12, no. 5, pp. 525-529, 2021. 
[22] B. A. Garibyan, "Determination of the Elastic Modulus of the Coating Using a Spherical Indenter," Turkish Journal of Computer and Mathematics Education (TURCOMAT), vol. 12, no. 10, pp. 1594-1600, 2021.

[23] M. O. Kaptakov, "Modelling of Mechanical Properties of Metal Plates with Polymer Coatings," Turkish Journal of Computer and Mathematics Education (TURCOMAT), vol. 12 , no. 5 , pp. 530-534, 2021.

[24]B. A. Garibyan, "Theoretical Estimations of Influence of Polymer Coatings on the Elastic Modulus and Ultimate Strength of Steel Samples," Turkish Journal of Computer and Mathematics Education (TURCOMAT), vol. 12, no. 10, pp. 1651-1656, 2021.

[25] M. O. Kaptakov, "Investigation of Effective Mechanical Characteristics of Nanomodified Carbon-Epoxide Composite by Numerical and Analytical Methods," Turkish Journal of Computer and Mathematics Education (TURCOMAT), vol. 12, no. 5, pp. 535-541, 2021.

[26] M. O. Kaptakov, "Obtaining of Carbon Fibers Based Composite Materials and Study of Their Mechanical Properties," Turkish Journal of Computer and Mathematics Education (TURCOMAT), vol. 12, no. 10, pp. 1601-1605, 2021.

[27] E. L. Kuznetsova, G. V. Fedotenkov, E. I. Starovoitov, "Methods of diagnostic of pipe mechanical damage using functional analysis, neural networks and method of finite elements," INCAS Bulletin, 12(Special Issue), pp. 79-90, 2020.

[28]N. A. Kucheva, V. Kohlert, "Analytical solution of the problem of thermoelasticity for a plate heated by a source with a constant heat supply on one surface," Turkish Journal of Computer and Mathematics Education (TURCOMAT), vol. 12, no. 10, pp. 1622-1633, 2021.

[29] E. L. Kuznetsova, A. V. Makarenko, "Mathematical model of energy efficiency of mechatronic modules and power sources for prospective mobile objects," Periodico Tche Quimica, 16 (32), pp. 529-541, 2016.

[30]L. N. Rabinskiy, E. L. Kuznetsova, "Analytical and numerical study of heat and mass transfer in composite materials on the basis of the solution of a stefan-type problem," Periodico Tche Quimica, 15 (Special Issue 1), pp. 339-347, 2018.

[31]N. A. Bulychev, E. L. Kuznetsova, "Ultrasonic Application of Nanostructured Coatings on Metals," Russian Engineering Research, 39 (9), pp. 809-812, 2019.

[32] N. A. Bulychev, V. V. Bodryshev, L. N. Rabinskiy, "Analysis of geometric characteristics of two-phase polymer-solvent systems during the separation of solutions according to the intensity of the image of micrographs," Periodico Tche Quimica, 16(32), pp. 551559, 2019.

[33] N. A. Kucheva, V. Kohlert, "Mathematical modeling methods for estimation the thermophysical properties of heat-protective composite materials," Turkish Journal of Computer and Mathematics Education (TURCOMAT), vol. 12, no. 10, pp. 1606-1612, 2021.

[34]N. A. Kucheva, "Investigation of the mechanical properties of heat-protective highly porous composite materials using the effective medium model," Turkish Journal of Computer and Mathematics Education (TURCOMAT), vol. 12, no. 10, pp. 1613-1621, 2021.

[35]L. N. Rabinskii, O. V. Tushavina, "Composite Heat Shields in Intense Energy Fluxes with Diffusion," Russian Engineering Research, 39(9), pp. 800-803, 2019.

[36] L. N. Rabinskiy, O. V. Tushavina, "Investigation of the influence of thermal and climate effects on the performance of tiled thermal protection of spacecraft," Periodico Tche Quimica, 16(33), pp. 657-667, 2019.

[37] V. V. Bodryshev, L. N. Rabinskiy, L. G. Nartova, N. P. Korzhov, "Geometry analysis of supersonic flow around two axially symmetrical bodies using the digital image processing method," Periódico Tchê Química, vol. 16, no. 33, pp. 541-548, 2019.

[38] N. A. Bulychev, M. A. Kazaryan, A. I. Erokhin, A. S. Averyushkin, L. N. Rabinskii, V. V. Bodryshev, B. A. Garibyan, "Analysis of the Structure of the Adsorbed Polymer Layers on the Surfaces of Russian Metallurgy (Metally)," vol. 2019, no. 13, pp. 1319-1325.

[39] O. A. Butusova, "Surface Modification of Titanium Dioxide Microparticles Under Ultrasonic Treatment," International Journal of Pharmaceutical Research, vol. 12, i. 4, pp. 2292-2296, 2020.

[40] O. A. Butusova, "Stabilization of Carbon Microparticles by High-Molecular Surfactants," International Journal of Pharmaceutical Research, vol. 12, Supplementary Issue 2, pp. 1147-1151, 2020.

[41] Yu. V. Ioni, A. Ethiraj, "New Tailor-Made Polymer Stabilizers for Aqueous Dispersions of Hydrophobic Carbon Nanoparticles," International Journal of Pharmaceutical Research, vol. 12, i. 4, pp. 3443-3446, 2020.

[42] Yu. V. Ioni, "Nanoparticles of noble metals on the surface of graphene flakes," Periodico Tche Quimica, vol. 17, no. 36, pp. 1199-1211, 2020.

[43] O. A. Butusova, "Vinyl Ether Copolymers as Stabilizers of Carbon Black Suspensions," International Journal of Pharmaceutical Research, vol. 12, Supplementary Issue 2, pp. 1152-1155, 2020.

[44] M. O. Kaptakov, "Catalytic Desulfuration of Oil Products under Ultrasonic Treatment," International Journal of Pharmaceutical Research, vol. 12, Supplementary Issue 2, pp. 1838-1843, 2020.

[45]B. A. Garibyan, "Enhancement of Mechanical Properties of Inorganic Glass under Ultrasonic Treatment," International Journal of Pharmaceutical Research, vol. 12, Supplementary Issue 2, pp. 1829-1832, 2020.

[46] M. O. Kaptakov, "Enhancement of Quality of Oil Products under Ultrasonic Treatment," International Journal of Pharmaceutical Research, vol. 12, Supplementary Issue 2, pp. 1851-1855, 2020.

[47] O. A. Butusova, "Adsorption Behaviour of Ethylhydroxyethyl Cellulose on the Surface of Microparticles of Titanium and Ferrous Oxides," International Journal of Pharmaceutical Research, vol. 12, Supplementary Issue 2, pp. 1156-1159, 2020. 
[48]A. N. Tarasova, "Vibration-based Method for Mechanochemical Coating Metallic Surfaces," International Journal of Pharmaceutical Research, vol. 12, Supplementary Issue 2, pp. 1160-1168, 2020.

[49]B. A. Garibyan, "Mechanical Properties of Electroconductive Ceramics," International Journal of Pharmaceutical Research, vol. 12, Supplementary Issue 2, pp. 1825-1828, 2020.

[50]M. O. Kaptakov, "Effect of Ultrasonic Treatment on Stability of $\mathrm{TiO}_{2}$ Aqueous Dispersions in Presence of Water-Soluble Polymers," International Journal of Pharmaceutical Research, vol. 12, Supplementary Issue 2, pp. 1821-1824, 2020.

[51] Yu. V. Ioni, "Synthesis of Metal Oxide Nanoparticles and Formation of Nanostructured Layers on Surfaces under Ultrasonic Vibrations," International Journal of Pharmaceutical Research, vol. 12, i. 4, pp. 3432-3435, 2020.

[52]A. N. Tarasova, "Effect of Reagent Concentrations on Equilibria in Water-Soluble Complexes," International Journal of Pharmaceutical Research, vol. 12, Supplementary Issue 2, pp. 1169-1172, 2020.

[53] G. A. Kalugina, A. V. Ryapukhin, "Impact of the 2020 Pandemic on Russian Aviation," Russian Engineering Research, vol. 41. no. 7, pp. 627-630, 2021.

[54]R. N. Zaripov, I. M. Murakaev, A. V. Ryapukhin, "Development of the Organization's Key Performance Indicators System in Order to Improve the Effectiveness of Its Human Capital and Risk Management," TEM Journal, vol. 10, no. 1, pp. 298-302, 2021.

[55]A. A. Kalugin, G. A. Kalugina, A. V. Ryapukhin, "Informational Support for the Sale of Passenger Aircraft," Russian Engineering Research, vol. 41, no. 2, pp. 183-187, 2021.

[56] R. N. Zaripov, I. M. Murakaev, S. V. Novikov, A. V. Ryapukhin, "Corporate Structure for Innovative Enterprises," Russian Engineering Research, vol. 40, no. 2, pp. 137-139, 2020.

[57]A. Y. Burova, "Concept of multistage discrete fourier transform without performing multiplications" Journal of Physics: Conference Series, vol. 1889, no. 2, 022003, 2021.

[58]A. Burova, "Reducing the Error of Digital Algorithms for Deductive Signal Processing Based on Their Multi-Stage Discrete Fourier Transform by the Difference Digital Filters," 22th International Conference on Digital Signal Processing and its Applications, DSPA 2020, no. 9213275, 2020.

[59] A. Y. Burova, "Minimization of asymmetry of thrust of dual-flow turbojet engines of airliner in accordance with the results of system analysis of thrust parameters," Asia Life Sciences, no. 2, pp. 629-643, 2019.

[60]A. N. Tarasova, "Effect of Vibration on Physical Properties of Polymeric Latexes," International Journal of Pharmaceutical Research, vol. 12, Supplementary Issue 2, pp. 1173-1180, 2020.

[61] Yu. V. Ioni, A. Ethiraj, "Study of Microparticles Surface Modification by Electrokinetic Potential Measuring,"
International Journal of Pharmaceutical Research, vol. 12, i. 4, pp. 3436-3439, 2020.

[62] Yu. V. Ioni, "Effect of Ultrasonic Treatment on Properties of Aqueous Dispersions of Inorganic and Organic Particles in Presence of Water-Soluble Polymers," International Journal of Pharmaceutical Research, vol. 12, i. 4, pp. 3440-3442, 2020.

[63]L. N. Rabinskiy, S. A. Sitnikov, "Development of technologies for obtaining composite material based on silicone binder for its further use in space electric rocket engines," Periodico Tche Quimica, 15(Special Issue 1), pp. 390-395, 2018.

[64]I. P. Lifanov, A. N. Astapov, V. S. Terentieva, "Deposition of heat-resistant coatings based on the $\mathrm{ZrSi} 2-$ MoSi2-ZrB2 system for protection of non-metallic composite materials in high-speed high-enthalpy gas flows," Journal of Physics: Conference Series, vol. 1713, no. 1, pp. 012025, 2020.

[65]I. P. Lifanov, A. A. Yurishcheva, A. N. Astapov, "Hightemperature protective coatings on carbon composites," Russian Engineering Research, vol. 39, no. 9, pp. 804 808, 2019.

[66] A. N. Astapov, I. P. Lifanov, M. V. Prokofiev, "Hightemperature interaction in the $\mathrm{ZrSi} 2-\mathrm{ZrSiO} 4$ system and its mechanism," Russian Metallurgy (Metally), no. 6, pp. $640-646,2019$.

\section{Sources of Funding for Research Presented in a Scientific Article or Scientific Article Itself}

This study was funded by RFBR, project number 20-0100523 .

\section{Creative Commons Attribution License $\mathbf{4 . 0}$ (Attribution 4.0 International, CC BY 4.0)}

This article is published under the terms of the Creative Commons Attribution License 4.0

https://creativecommons.org/licenses/by/4.0/deed.en US 